\title{
Neuer Antikörper bessert eosinophiles Asthma
}

\section{Asthmapatienten mit erhöhten Eosinophilen-Zahlen werden einer Phase-II- Studie zufolge durch den Antikörper Dupilumab vor Exazerbationen ge- schützt. Der Antikörper unterbindet die Wirkung von Interleukin-4 und -13.}

— rhöhte Spiegel von eosinophilen Gra_ nulozyten sind ein Indikator für eine von Typ-2-T-Helferzellen (Th2) dominierte Immunantwort. Th2-assoziierte Zytokine, insbesondere Interleukin(IL)-4 und -13 , spielen auch bei Asthma eine Rolle. Mit dem humanen monoklonalen Antikörper Dupilumab, der sich gegen die Alfa-Untereinheit des IL-4-Rezeptors richtet, kann die Wirkung dieser beiden Zytokine blockiert werden.

Der Antikörper wurde nun in einer Phase-II-Studie bei Asthmapatienten mit erhöhten Eosinophilen-Konzentrationen im Blut $(\geq 300 / \mathrm{ml})$ oder im Sputum $(\geq$ $3 \%)$ getestet. Alle Patienten litten an persistierendem Asthma, das trotz inhalierbarer Glukokortikoide in Kombination mit lang wirksamen Betamimetika nicht unter Kontrolle zu bringen war. Jeweils 52 Patienten wurden maximal zwölf Wochen lang mit Dupilumab (1 x pro Woche $300 \mathrm{mg}$ s.c.) bzw. Placebo behandelt. Nach vier Wochen sollten die Patienten das Betamimetikum absetzen und in den Wochen sechs bis neun auch das Glukokortikoid ausschleichen.

Asthmaexazerbationen, der primäre Studienendpunkt, traten daraufhin bei drei Verumpatienten (6\%) und 23 Placebopatienten ( $44 \%$ ) auf; das entsprach einer Risikoreduktion um $87 \%$ mit Dupilumab ( $\mathrm{p}<0,001)$. Unter dem Antikörper kam es gegenüber dem Ausgangswert zu einer signifikanten Zunahme der Einsekundenkapazität (FEV1), die trotz Absetzens der Basistherapie bis Woche zwölf erhalten blieb. Auch beim exspiratori- schen Spitzenfluss und bei der Asthmakontrolle wurden anhaltende Verbesserungen festgestellt. Gleichzeitig sank die Konzentration von mehreren Biomarkern einer Th2-vermittelten Entzündung.

Nebenwirkungen waren mit Dupilumab ähnlich häufig wie mit Placebo und im Allgemeinen leicht bis mittelschwer. Meistens handelte es sich um Reaktionen an der Einstichstelle, Nasopharyngitiden, Übelkeit und Kopfschmerzen. Eine Patientin entwickelte ein Angioödem, das zum Therapieabbruch zwang.

Fazit: Bei Patienten mit mittelschwerem bis schwerem Asthma und erhöhter Zahl an Eosinophilen führt das Absetzen der Basistherapie mit inhalativen Glukokortikoiden und lang wirksamen Betamimetika seltener zu Exazerbationen, wenn sie den Antikörper Dupilumab erhalten.

Dr. Beate Schumacher

Wenzel S et al. Dupilumab in Persistent Asthma with Elevated Eosinophil Levels. N Engl J Med 2013; 368: 2455-66

\section{Springer Medizin}

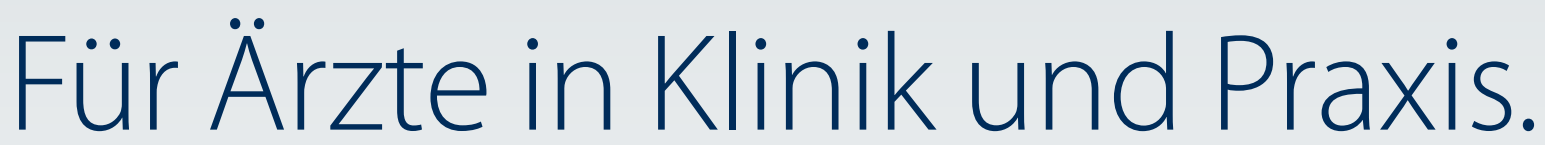

- Neueste Antibiotika/ Antimykotika, neueste Resistenzen

- Nach Substanzen, Erregern und Indiekationen geordnete Tabellen

16. A. 2012. Etwa 270 S. Brosch.

- Ca. $€(D) 22,95$

$€$ (A) $23,59 \mid \mathrm{sFr} 29,00$ ISBN 978-3-642-25678-3

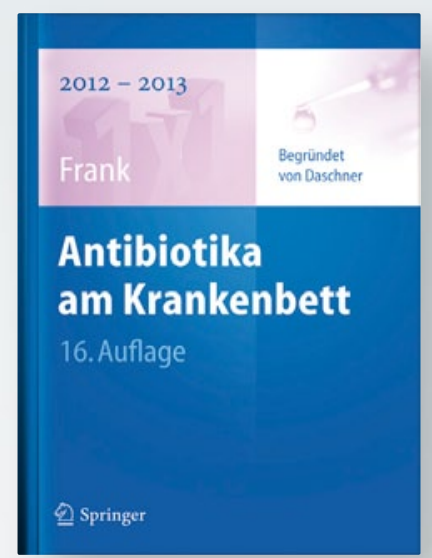

- Die wichtigsten Antibiotika und Infektionen

- Umweltschonende und Kosten sparende Hygiene-Tipps

- Neueste Antibiotika/ Antimykotika, neueste Resistenzen

10. A. 2012. Etwa 200 S. Brosch.

- Ca. $€$ (D) 22,95

$\in($ A) $23,59 \mid$ sFr 29,00

ISBN 978-3-642-25626-4

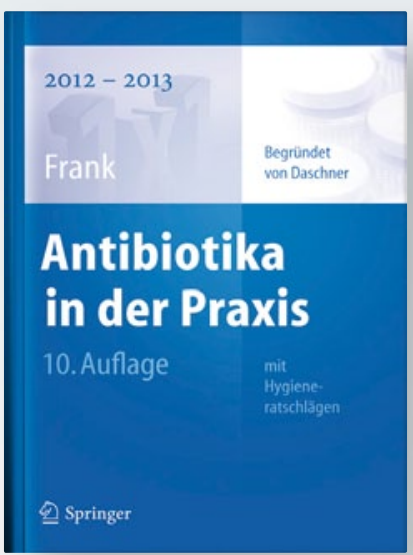

